\title{
Centered-gap and aligned-gap multiple split ring resonator for bio-sensing application
}

\begin{abstract}
In general, a classical Split Ring Resonator (SRR) structure exhibits high Q-factor based on deeper and sharper transmission dips at resonance as well as produce high electric field density at the gaps. It is believed, by introducing more gaps, a strong and localized E-field will be obtained in the area between the split gaps. Based on these features, three types of rectangular multiple Split Ring Resonators (SRRs) were proposed to resonate in the frequency range of 3-7 GHz and simulated using Computer Simulation Technology (CST) Microwave Studio to determine the transmission characteristics and the resonance frequency. A Nicolson-Ross-Weir (NRW) technique is used to retrieve the effective parameters from the resultant S-parameter. It is shown that the resonance frequency of investigated structures falls in a frequency region in which the real part of permeability is negative. Later, the simulated results were investigated and the performances as well as the size of each unit cell itself were compared. Simulation for three different type of dielectric samples were also presented to demonstrate that the proposed structure may be well suited for bio-sensing.
\end{abstract}

Keyword: Metamaterials; Split ring resonator; Negative permeabilty; Q-factor 